\title{
Now you see it, Now you don't: compulsive exercise in adolescents with an eating disorder
}

\author{
Johanna Levallius ${ }^{1,2^{*}}$ (D), Christina Collin ${ }^{1,2}$ and Andreas Birgegård ${ }^{1,2}$
}

\begin{abstract}
Background: Compulsive exercise (CE) has been proposed as significant in the etiology, development and maintenance of eating disorders (EDs), resulting in more severe and enduring pathology. However, few studies have investigated CE longitudinally in adolescents with EDs. We aimed to test if adolescents show the same associations between CE and other clinical variables as previous research has found in adults.
\end{abstract}

Methods: Three thousand one hundred sixteen girls and 139 boys from a clinical ED database were investigated regarding prevalence and frequency of CE and its relation to psychiatric symptoms, associated features and outcome. Denial of illness is common among adolescents and was therefore adjusted for.

Results: Adjusted CE prevalence in girls was 44\%, and CE was most prevalent in bulimia nervosa. As previously found in adults, those with CE scored significantly higher than non-CE on total ED severity, level of restriction and negative perfectionism. However, there were only minor differences between CE and non-CE patients on emotional distress, hyperactivity, suicidality and self-esteem. Among boys, adjusted CE prevalence was 38\%, and CE boys scored significantly higher than non-CE on total ED severity. Initial CE did not influence 1-year outcome, although cessation of CE was associated with remission.

Conclusions: CE is a common clinical feature in adolescents with EDs and cessation is associated with remission. When controlling for denial of illness, CE had less detrimental impact than predicted. We recommend controlling for denial in studies on ED adolescents and further exploration of classification and treatment implications of CE.

Keywords: Eating disorder, Compulsive exercise, Compulsive exercise, Behavioral addiction, Adolescents, Denial

\section{Plain English Summary}

The aims were to investigate how compulsive exercise (CE) related to eating disorder (ED) symptoms and outcome in adolescent girls and boys with EDs. We used a nationwide clinical ED database of 3116 girls and 139 boys. Forty-four percent of adolescents reported engaging in $C E$ on average 3.9 times per week $(S D=2.6)$. Patients reporting $C E$ had a slightly more severe clinical presentation. At 1 year, 55\% had remitted from ED and prognosis was not affected by initial CE. However, cessation of CE increased the likelihood of remission from ED.

\footnotetext{
* Correspondence: johanna.levallius@ki.se

1 Department of Clinical Neuroscience, Resource Center for Eating Disorders, Center for Psychiatry Research, Karolinska Institutet, SE-113 64 Stockholm, Sweden

${ }^{2}$ Resource Center for Eating Disorders, Stockholm Health Care Services, Stockholm County Council, SE-113 64 Stockholm, Sweden
}

\section{Background}

Physical exercise is regarded by both society and health care as a healthy way to diminish risk for, and detrimental effects of, several common diseases. However, from an eating disorders (EDs) perspective, exercise can be highly problematic. Compulsive exercise (CE) has been recognized as significant in the etiology, development, and maintenance of EDs [1-4], and is generally associated with more severe [5-7] and enduring ED pathology [8-10]. Both CE and EDs show detrimental effects on healthrelated quality of life, and interact to lessen quality of life further [11]. Moreover, rigorous exercise to control weight and/or shape has been linked to increased suicidality over and above other ED symptoms in both clinical and nonclinical adult samples [12]. EDs in themselves are serious illnesses and the co-occurrence of CE seems to exacerbate 
the condition, yet there is very limited research investigating this in adolescents. The current study therefore aimed to investigate the longitudinal relationship between different EDs and CE in the largest adolescent sample to date.

The terminology to describe problematic exercise has varied in the literature. The term compulsive exercise will be used here, as it is the most established term [13]. In an ED context, "CE describes a condition of weight and shape concern and a persistent continuation of exercise in order to (a) mitigate guilt/negative affect if not exercising, and (b) avoid perceived negative consequences of stopping" ([1], $p$ 184). The two largest studies investigating CE among adults with EDs estimate a prevalence of $36-45 \%$, varying depending on diagnostic subgroup and $\mathrm{CE}$ definition $[6,14]$. The important factor seems to be presence or absence of CE rather than frequency, although CE among patients is more frequent than among healthy controls (9.9 vs 1.6 in 28 days) [15]. The few studies including adolescents found no difference compared to adults in prevalence or frequency of $\mathrm{CE}[4,16]$, but a recent review found an average prevalence in studies of $\mathrm{CE}$ among adolescents with ED to be 52.6\% [17]. In studies on adults, ED onset occurred at an earlier age in CE patients [6], who also showed more associated features such as perfectionism [6, 18], obsessive/compulsive behavior [19], and hyperactivity [16]. ED is consistently associated with low self-esteem, but whether patients with $\mathrm{CE}$ have even more impaired self-esteem is uncertain $[20,21]$. Findings regarding whether $\mathrm{CE}$ is associated with depression and anxiety are also inconsistent $[1,17,21]$.

Research findings detailed above pertain to females, as research on males with EDs and CE is scarce. Welch and colleagues [14] found comparable CE levels in men with ED as in women, despite lower ED symptomatology in men. In both sexes, exercise frequency has been linked to body dissatisfaction [22]. A large study on prediction of compulsive exercise in 12-14 year old normal boys and girls found the same three major predictors in both groups: drive for thinness, self-directed perfectionism and obsessive-compulsiveness [23].

Two aspects should be taken into account when examining exercise in EDs; one quantitative (frequency, duration, intensity), the other qualitative (compulsivity and rigidity) [24]. Several validated measures have been developed to capture various dimensions of these two aspects [13]. In this study, presence of $\mathrm{CE}$ was determined by a question in the Eating Disorder Examination Questionnaire (EDEQ), not originally designed to capture CE. However, studies have found strong correlations between this item and established CE measures [12, 13], supporting the use of EDEQ items as an approximation of CE.

Another factor to be taken into account is that minimizing or denying symptoms is common in adolescents with ED [25], possibly affecting the validity of symptom assessment. We therefore sought to explore this particular feature as a possible confounder, under the umbrella term "denial of illness".

In summary, based on the studies reviewed above, our aims were to investigate how $\mathrm{CE}$ relates to ED diagnosis, symptoms, associated features and outcome in adolescents with ED, using a nationwide clinical database. Based on previous findings in adults, we expected similar CE prevalence in adolescents as in adults, and that CE would be associated with earlier ED onset, higher symptomatology, suicidality, negative perfectionism, hyperactivity, and a poorer prognosis. We did not expect effects of CE frequency or sex differences. No hypotheses were posited regarding $\mathrm{CE}$ and its relation to depression or self-esteem. In addition, we investigated the potential effect of denial of illness, a problematic feature particularly common in adolescents with ED.

\section{Methods \\ Participants and procedure}

Data came from the Stepwise database, a nationwide Swedish longitudinal clinical database of ED patients seeking treatment at specialist psychiatric treatment units [26]. All patients aged 13-17 registered in the database from 2005-03-07 (start of the database) through 2015-06-16 were extracted $(N=3982)$. Inclusion criteria were medical or self-referral to one of the participating treatment units, a diagnosed DSM-IV ED, and consent to research use of data. Three thousand two hundred and fifty-five patients fulfilled inclusion criteria, and $95.7 \%$ were girls. Diagnoses were anorexia nervosa (AN), bulimia nervosa $(\mathrm{BN})$ and ED not otherwise specified (EDNOS). Patients received treatment of various modalities (e.g., medical, psychological, pedagogical, nutritional, social, physical), length, and intensity (e.g., residential, day and/or out-patient treatment).

During the time of data collection, between 60 and 90\% of specialist units participated, and coverage ratio within units was high (>90\%). Stepwise assessment, including interviews and self-report questionnaires, was performed within the first three visits (for inpatients, within the first week of care), and self-report instruments had satisfactory psychometric properties. Age at onset was defined as the patient's retrospective report of symptom debut. Remission was defined as not fulfilling any ED diagnosis according to the semi-structured EDs interview described below. Ethical approval was granted by the regional review board (No. 2009/1298-31/1, EPN Stockholm).

\section{Measures}

The Structured Eating Disorder Interview (SEDI) is a semi-structured computerized interview with 20-30 questions, used to assess DSM-IV diagnostic criteria, with number of questions automatically varying depending on 
presenting symptoms [27]. BMI cutoffs defining underweight for AN were adjusted for age and sex, based on Swedish norms. Preliminary validation against the EDE interview has shown a concordance of $81 \%$ regarding specific ED diagnosis (including EDNOS and BED) and Kendall's tau-b of $0.69(p<.001)$ [27].

The Eating Disorder Examination Questionnaire (EDEQ, adolescent version) [28] estimated ED symptoms. EDEQ consists of 36 items, 23 of them rated on a seven degree scale. Scores on items are averaged into four subscales; restraint, eating concern, weight concern, and shape concern, averaged into a global score. Thirteen items cover presence and frequency of binge eating, self-induced purging, and compulsive exercise. Patients were divided into CE or noncompulsive exercisers (non-CE) by the dichotomous "Have you exercised hard to control weight or shape?". This was followed by a CE-frequency question, pertaining to the last 14 days. The EQEQ for adolescents has failed to reproduce the original four factor structure [29]. However, for the purpose of this study we only included frequency items, the restraint subscale and the global score, which have shown satisfactory psychometric properties [29].

Mini International Neuropsychiatric Interview for Children and Adolescents (MINI-Kid) is a psychometrically sound semi-structured interview of Axis-I disorders, performed by clinicians consisting of several modules. Here we used data from the A module, which assesses caseness of depression, and the B module, which assesses suicidality, the latter both as presence/absence and a three-stage level of risk [30].

The Strengths and Difficulties Questionnaire (SDQ) measures self-rated behavioral and psychological problems among children and adolescents in 25 items rated on a $3^{\circ}$ scale covering emotional distress, behavioral problems, hyperactivity/inattention, and problems with peers. It also includes a fifth positive scale, prosocial behavior. Based on the theoretical aims, the subscales emotional distress and hyperactivity/inattention were used. The SDQ has acceptable internal consistency, test-retest stability, and content validity [31].

The Structural Analysis of Social Behaviour (SASB) measures self-image in 36 items, with statements rated on a 0-100 (10-point increment) scale [32]. The SASB model is a circumplex organized around two orthogonal dimensions: the horizontal Affiliation axis, ranging from self-love to self-hate/self-hostility, and the vertical Autonomy axis, ranging from self-control to spontaneity/letting go. Matching our aims, we selected two variables: the Affiliation vector (computed as a weighted summary score for items pertaining to the Affiliation axis [32] that has been shown to approximate self-esteem [33]), and Self-criticism, involving self-blame over mistakes, selfaccusation, and negative comparisons to others, which approximates negative perfectionism.

\section{Statistical analysis}

Analyses were carried out using SPSS Version 22. Independent two-tailed $t$-tests were used for comparisons between CE and non-CE. Cohen's $d$ effect size was assessed according to small $(\geq .20)$, medium $(\geq .50)$, and large $(\geq .80)$ conventions. Chi-square test was used to test for differences in proportion of CE patients by diagnostic subgroup, depression and suicide risk. Pearson correlations were computed to investigate the relationship between the different variables and CE frequency, while linear regression was used to test if $\mathrm{CE}$ frequency predicted severity at 1 year. Logistic regression was used to test if initial presence of $\mathrm{CE}$ could predict remission. Alpha was set at $p \leq .001$ for the full subsample of girls due to the large $n$, and $p \leq .05$ for boys as well as for girls when divided into diagnostic ED subcategories. To adjust for outliers, a maximum CE frequency of 30 in the last 2 weeks was set $(<0.1 \%$ of ratings). Denial of illness was defined as scoring below age-, sex-, and diagnosis-adjusted clinical cutoff on the EDEQ global score, using Clinical Significance (CS) calculations as described in Ekeroth and Birgegård [34].

\section{Results}

To first address denial of illness, we explored the proportion scoring below EDEQ clinical range. 25\% of adolescents with EDs rated below clinical cut-off; this was most common among AN patients (31\% of AN patients denied illness). Patients below cut-off were termed 'deniers' and those above 'clinical'. Deniers generally rated lower on all psychopathology, including CE, where prevalence was $12 \%$ versus $44 \%$ for the clinical group. Further analyses were therefore performed in two ways: for all patients and for clinical patients only.

\section{Girls}

CE proportion for all girls was $36 \%$, and mean frequency was 3.9 times/week $(S D=2.62)$. For all girls and the clinical group respectively, CE prevalence differed significantly by diagnosis $\left(\chi^{2}(2,3116)=88.813, p<.001\right.$, and $\left.\chi^{2}(2,2345)=49.120, p<.001\right)$, and was most prevalent in $\mathrm{BN}$ (Table 1). Excluding deniers increased overall prevalence to $44 \%$, with the largest increases in AN and EDNOS.

Independent $t$-tests showed that $\mathrm{CE}$ patients differed significantly from non-CE patients on several measures: global EDEQ, restraint, negative perfectionism, emotional distress, hyperactivity, and self-esteem (Table 2). However, exclusion of deniers reduced effect sizes considerably. Fifty percent were diagnosed with depression in the clinical group ( $41 \%$ for all girls), with chi-square test finding no significant difference in prevalence of depression between CE and non-CE patients $\left(\chi^{2}(1,2170)=1.083, p=.30\right)$. Contrary to hypothesis, $\mathrm{CE}$ was not associated with suicide 
Table 1 Group characteristics by diagnosis in girls

\begin{tabular}{|c|c|c|c|c|c|c|c|c|}
\hline \multicolumn{9}{|l|}{ All girls } \\
\hline & AN & $(S D)$ & $\mathrm{BN}$ & $(S D)$ & EDNOS & $(S D)$ & Total & $(S D)$ \\
\hline$n$ & 1112 & & 329 & & 1675 & & 3116 & \\
\hline Age & 15.1 & (1.35) & 16.2 & $(1.04)$ & 15.4 & (1.33) & 15.4 & (1.34) \\
\hline Body mass index & 15.8 & $(1.42)$ & 22.0 & $(3.91)$ & 19.2 & (2.97) & 18.3 & (3.35) \\
\hline EDEQ global & 3.1 & $(1.60)$ & 4.2 & $(1.08)$ & 3.4 & (1.53) & 3.39 & (1.55) \\
\hline CE (\%) & 26 & & 50 & & 40 & & 36 & \\
\hline CE frequency & 7.4 & (6.5) & 6.3 & (3.9) & 7.2 & $(5.2)$ & 7.1 & (5.4) \\
\hline \multicolumn{9}{|l|}{ Clinical group } \\
\hline$n$ & 772 & & 306 & & 1267 & & 2345 & \\
\hline Age & 15.1 & (1.35) & 16.2 & $(1.06)$ & 15.4 & $(1.31)$ & 15.4 & (1.33) \\
\hline Body mass index & 15.9 & (1.41) & 22.0 & $(3.79)$ & 19.5 & (2.95) & 18.6 & (3.40) \\
\hline EDEQ global & 4.0 & $(1.01)$ & 4.4 & $(0.79)$ & 4.2 & $(0.86)$ & 4.13 & $(0.91)$ \\
\hline CE (\%) & 34 & & 53 & & 48 & & 44 & \\
\hline CE frequency & 8.5 & (6.4) & 6.7 & (3.7) & 8.0 & (5.1) & 7.9 & (5.3) \\
\hline
\end{tabular}

CE frequency reported for last 14 days

Note: $A N$ anorexia nervosa, $B N$ bulimia nervosa, $E D N O S$ eating disorder not otherwise specified, $B M I$ body mass index, EDEQ eating disorder examination questionnaire, $C E$ compulsive exercise

risk $\left(\chi^{2}(1,2063)=0.018, p=.89\right)$. CE frequency correlated significantly but weakly with EDEQ global $(r=.176)$, restraint $(r=.161)$, binge frequency $(r=.248)$, negative perfectionism $(r=.100)$, and hyperactivity $(r=.124)$.

The characteristics of CE patients might differ depending on ED diagnosis. The pairwise comparisons above were therefore repeated for the three diagnostic subgroups, excluding deniers. As depicted in Table 3, CE patients were highly similar to each other on all variables investigated, regardless of ED diagnosis. When looking at pairs within each diagnosis, differences emerged mainly for AN patients, where CE patients scored higher than
non-CE on ED symptomatology, negative perfectionism, and self-esteem. Chi-square tests showed no significant difference in prevalence of depression between pairs $\left(\mathrm{AN} \mathrm{X}^{2}(1,723)=1.212, p=.15, \mathrm{BN}^{2}(1,285)=\right.$ $\left.0.732, p=.23 \operatorname{EDNOS} \chi^{2}(1,1162)=0.754, p=.21\right)$, yet suicide risk was significantly more prevalent in AN with $\mathrm{CE}\left(\mathrm{AN} \chi^{2}(1,678)=6.471, p=.01, \mathrm{BN} \mathrm{X}^{2}(1,274)=\right.$ $0.000, p=0.55$ EDNOS $\left.\chi^{2}(1,1111)=2.734, p=.06\right)$.

One-year follow-up data was available for $52 \%$ of the sample. There was a significant overall decrease in global EDEQ score $\left(M_{\text {diff }}=1.90, t=32.38, p<.001\right)$ and the majority (55\%) had also remitted from ED. CE patients

Table 2 Group comparisons of symptomatology in CE vs. non-CE female patients

\begin{tabular}{|c|c|c|c|c|c|c|c|c|}
\hline & \multicolumn{4}{|l|}{ All girls } & \multicolumn{4}{|c|}{ Clinical group } \\
\hline & CE & Non-CE & $t$ & $d$ & CE & Non-CE & $t$ & $d$ \\
\hline Age at onset & $13.8(1.7)$ & $13.7(1.9)$ & 0.03 & - & $13.7(1.7)$ & $13.7(1.9)$ & 0.56 & - \\
\hline \multicolumn{9}{|l|}{ EDEQ } \\
\hline Global & $4.1(1.1)$ & $3.0(1.6)$ & $23.61^{*}$ & 0.87 & $4.4(0.9)$ & $4.0(0.9)$ & $10.85^{*}$ & 0.46 \\
\hline Restraint & $4.1(1.4)$ & $2.7(1.9)$ & $22.82^{*}$ & 0.86 & $4.3(1.3)$ & $3.7(1.4)$ & $10.60^{*}$ & 0.44 \\
\hline Binge $(M)$ & $7.6(10.1)$ & $7.7(10.8)$ & 0.20 & - & $7.8(10.4)$ & $8.5(11.2)$ & 1.11 & - \\
\hline Purge $(M)$ & $7.6(9.1)$ & $8.3(9.7)$ & 1.01 & - & $7.8(9.2)$ & $8.6(10.0)$ & 1.21 & - \\
\hline \multicolumn{9}{|l|}{ SASB } \\
\hline Negative perfectionism & $58.7(23.7)$ & $44.7(25.9)$ & $15.34^{*}$ & 0.56 & $61.1(22.4)$ & $55.7(21.9)$ & $5.94^{*}$ & 0.25 \\
\hline Self-esteem & $-13.9(35.2)$ & $6.1(41.2)$ & $14.26^{*}$ & 0.56 & $-18.0(32.5)$ & $-12.2(32.9)$ & $4.28^{*}$ & 0.18 \\
\hline \multicolumn{9}{|l|}{ SDQ } \\
\hline Emotional distress & $6.2(2.3)$ & $5.3(2.6)$ & $10.42^{*}$ & 0.41 & $6.5(2.1)$ & $6.3(2.1)$ & 1.91 & - \\
\hline Hyperactivity & $4.9(2.3)$ & $4.1(2.4)$ & $9.68^{*}$ & 0.36 & $5.0(3.3)$ & $4.6(2.3)$ & $3.88^{*}$ & 0.16 \\
\hline
\end{tabular}


Table 3 Symptomatology in clinical girls with CE vs. non-CE by ED diagnosis

\begin{tabular}{|c|c|c|c|c|c|c|c|c|c|c|c|c|}
\hline & \multicolumn{4}{|l|}{ AN } & \multicolumn{4}{|l|}{$\mathrm{BN}$} & \multicolumn{4}{|l|}{ EDNOS } \\
\hline & $\mathrm{CE}$ & Non-CE & $t$ & $d$ & CE & Non-CE & $t$ & d & CE & Non-CE & $t$ & $d$ \\
\hline Onset & $13.4(1.8)$ & $13.6(1.8)$ & 0.98 & - & $13.7(1.8)$ & $13.1(2.9)$ & 1.24 & - & $13.7(1.7)$ & $13.7(2.1)$ & 0.44 & - \\
\hline \multicolumn{13}{|l|}{ EDEQ } \\
\hline Global & $4.3(1.0)$ & $3.8(1.0)$ & $6.56^{*}$ & 0.50 & $4.5(0.8)$ & $4.3(0.82)$ & 2.32 & - & $4.3(0.8)$ & $4.0(0.9)$ & $7.28^{*}$ & 0.41 \\
\hline Restraint & $4.4(1.3)$ & $3.7(1.4)$ & $6.92^{*}$ & 0.52 & $4.1(1.2)$ & $3.8(1.43)$ & 1.97 & - & $4.3(1.2)$ & $3.7(1.4)$ & $7.85^{*}$ & 0.44 \\
\hline \multicolumn{13}{|l|}{ SASB } \\
\hline Negative perfectionism & $63.4(22.3)$ & $52.9(23.4)$ & $6.00^{*}$ & 0.45 & $63.2(21.1)$ & $61.0(21.5)$ & 0.89 & - & $59.6(22.6)$ & $56.7(20.4)$ & 2.44 & - \\
\hline Self-esteem & $-20.0(34.3)$ & $-8.5(34.8)$ & $4.39^{*}$ & 0.33 & $-20.5(29.2)$ & $-19.8(32.1)$ & 0.20 & - & $-16.4(32.5)$ & $-13.3(31.3)$ & 1.74 & - \\
\hline \multicolumn{13}{|l|}{ SDQ } \\
\hline Emotional distress & $6.5(2.2)$ & $6.0(2.2)$ & 2.89 & - & $6.5(2.1)$ & $6.5(2.1)$ & 0.16 & - & $6.5(2.1)$ & $6.4(2.1)$ & 0.12 & - \\
\hline Hyperactivity & $5.0(2.3)$ & $4.4(2.2)$ & 3.47 & - & $5.1(2.2)$ & $5.4(2.4)$ & 0.92 & - & $5.0(2.4)$ & $4.6(2.3)$ & 2.53 & - \\
\hline
\end{tabular}

Note: See Table 2 for explanation of abbreviations

${ }^{*}=p<0.001$

were hypothesized to have a poorer remission rate and this was disconfirmed, both for all girls and the clinical group $X^{2}(1,1059)=2.450, p=.12$ and $\chi^{2}(1,825)=$ $0.627, p=.43$. CE frequency at baseline did not predict either remission (logistic regression $\chi^{2}(1,399)=1.77$, $p=.18)$ or symptom improvement as measured by EDEQ (linear regression $F[1,381]=.081, p=.78$ ). At follow-up, there was a certain degree of cross-over, meaning most patients ceased to utilize CE as compensatory behavior (69\% of CE patients) whereas others began (17\% of non-CE) (Table 4). Those that ceased with $\mathrm{CE}$ had a greater likelihood of remission compared to those who continued $\left(\chi^{2}(1,381)=22.33, p<.001\right)$, see Table 4.

\section{Boys}

Twenty-nine percent of boys reported CE (38\% in the clinical group), with a mean frequency of 4.1 times/week $(S D=2.83)$, see Table 5. As with CE girls, CE boys had significantly higher global EDEQ and restraint score, and reported more negative perfectionism, emotional distress, and hyperactivity (Table 6). Performing analyses again after excluding deniers (35 CE and 57 non-CE remaining), effect sizes were reduced even more than for girls. The only significant difference remaining was that CE boys had higher Global EDEQ than non-CE boys. Twenty-eight

Table 4 Remission rate of clinical girls by CE status at baseline and follow-up $(n=779)$

\begin{tabular}{llllll}
\hline $\begin{array}{l}\text { Distribution } \\
\text { of patients }\end{array}$ & $\begin{array}{l}\text { CE at } \\
\text { baseline }\end{array}$ & $\begin{array}{l}\text { CE at } \\
1 \text { year }\end{array}$ & $\begin{array}{l}\text { EDEQ } \\
\text { baseline }\end{array}$ & $\begin{array}{l}\text { EDEQ } \\
1 \text { year }\end{array}$ & $\begin{array}{l}\text { Remission } \\
\text { rate }\end{array}$ \\
\hline $45 \%$ & No & No & $3.95(0.88)$ & $1.74(1.47)$ & $56 \%$ \\
$32 \%$ & Yes & No & $4.22(0.89)$ & $1.72(1.50)$ & $59 \%$ \\
$14 \%$ & Yes & Yes & $4.43(0.78)$ & $3.61(1.55)$ & $29 \%$ \\
$9 \%$ & No & Yes & $4.15(0.85)$ & $3.47(1.42)$ & $39 \%$ \\
\hline
\end{tabular}

Note: $C E$ compulsive exercise, EDEQ eating disorder examination questionnaire percent of boys had depression, with higher prevalence in the CE group $(41 \%)\left(\chi^{2}(1,130)=4.947, p=.03\right)$, but $C E$ was not associated with suicide risk $\left(\chi^{2}(1,119)=0.584\right.$, $p=.45)$. CE frequency did not correlate significantly with any variables in Table $6(r$ range $=-.155-.257)$. Sample size did not permit analyses by ED subgroup.

At 1-year follow-up, $58 \%$ of boys had remitted, and initial CE status did not affect prognosis $\left(\chi^{2}(1,45)\right.$ $=.046, p=0.55)$ for boys either. Overall, there was a significant decrease in global EDEQ score $\left(M_{\text {diff }}=1.37\right.$, $p<.001)$, where an independent $t$-test showed that $\mathrm{CE}$ patients again did not differ from non-CE patients $(t=0.214, p=.83)$ at 1 -year.

\section{Discussion}

This is the largest longitudinal study to date investigating presence of $\mathrm{CE}$ in adolescents with EDs. We found comparable CE prevalence among adolescents (44\%) as previous research has reported in adults $[6,14]$, and comparable also to previous average estimates in ED adolescents [17]. Among girls, CE was more common in $\mathrm{BN}$ and EDNOS than in AN, a pattern also found in a previous representative Swedish study on adults [14]. After controlling for denial of illness, there was less difference between $\mathrm{CE}$ and non-CE patients on symptomatology and associated features than findings on adults would suggest [1, 16-21]. The study also included a sizeable number of boys, presenting the same general pattern as girls; however not all comparisons could be carried out for boys due to sample size. Overall, and contrary to studies on adults [8-10], CE adolescents did not have a worse prognosis in comparison to non-CE adolescents, provided they ceased with CE. At 1 year follow-up, twothirds of adolescents reporting $\mathrm{CE}$ had ceased doing so, and remission rate was similar for them as for non-CE adolescents (59\% vs. 56\%). Adolescents still reporting CE 
Table 5 Group characteristics by diagnosis in boys

\begin{tabular}{lllllll}
\hline & AN & (SD) & EDNOS & (SD) & Total & (SD) \\
\hline All boys & & & & & & \\
$n$ & 40 & & 96 & & 139 & \\
Age & 15.0 & $(1.35)$ & 15.2 & $(1.33)$ & 15.1 & $(1.33)$ \\
Body mass index & 16.1 & $(1.42)$ & 19.8 & $(2.97)$ & 18.8 & $(3.68)$ \\
EDEQ global & 2.1 & $(1.60)$ & 2.1 & $(1.53)$ & 2.2 & $(1.62)$ \\
CE (\%) & 25 & & 29 & & 29 & \\
CE Frequency & 6.5 & $(3.78)$ & 8.4 & $(6.44)$ & 7.9 & $(5.81)$ \\
Clinical group & & & & & & \\
$n$ & 24 & & 65 & & 92 & \\
Age & 15.0 & $(1.16)$ & 15.1 & $(1.43)$ & 15.1 & $(1.34)$ \\
Body mass index & 16.2 & $(1.07)$ & 20.4 & $(4.07)$ & 19.4 & $(4.08)$ \\
EDEQ global & 3.3 & $(1.23)$ & 2.9 & $(1.19)$ & 3.1 & $(1.24)$ \\
CE (\%) & 38 & & 39 & & 38 & \\
CE Frequency & 5.6 & $(3.36)$ & 7.7 & $(5.05)$ & 7.1 & $(4.48)$ \\
\hline
\end{tabular}

Note: See Table 1 for list of abbreviations. Boys with bulimia nervosa omitted $(n=3)$

at follow-up had lower remission rate (29\%), as did those who had begun with CE during treatment (39\%). The general finding in the current study of weaker associations than expected between $\mathrm{CE}$ and ED features might be due to a preponderance of inpatients and AN patients in studies (e.g., [8-10, 13, 16, 19-21]), where findings might not be generalizable to ED patients.

CE girls and boys alike rated higher ED symptomatology, in line with numerous previous studies on female adults [5-7]. At the subgroup level, CE was associated with higher negative perfectionism in AN girls, due to non-CE girls with AN actually rating a lesser degree of negative perfectionism than $\mathrm{BN}$ and EDNOS. CE was also associated with higher frequency of suicidality in AN girls; paradoxically not accompanied by elevated emotional distress or depression. Considering an elevated suicide risk in AN patients [35] in general, this merits further investigation. $\mathrm{CE}$ was associated with elevated ED symptom levels in AN and EDNOS, but not in BN. CE was furthermore not associated with depression, selfesteem (except in clinical AN) or hyperactivity, and it is not clear if $\mathrm{CE}$ itself may have buffered these factors, by mitigating negative affect [36], shame [37], and excess energy levels [16], or if CE is unrelated to them. Lastly, in line with our hypothesis, CE frequency had little impact on investigated associations, even though frequency ranged from twice a day to once biweekly.

\section{Theoretical and treatment implications}

The variety of definitions and assessment methods for $\mathrm{CE}$ poses challenges in comparing studies. One other large scale study on a representative sample of adults has used EDEQ items [14], corroborating our estimates for adolescents. However, estimating CE by two self-rated EDEQ items has limitations. Despite being the most established ED measure, it does not capture all aspects of $\mathrm{CE}$. Evidence however suggests that the EDEQ does capture the global concept fairly well, having a correlation of .64 with the Compulsive Exercise Test [13] and .57 with the Obligatory Exercise Questionnaire [12]. Thus, the EDEQ may profitably be used as a preliminary indication of $\mathrm{CE}$, to then be assessed in greater detail with measures specifically designed to do so. Furthermore, given the high prevalence of CE in EDs, and its possible detrimental impact, perhaps CE has earned DSM criterion status in ED subcategories other than $\mathrm{BN}$, where it is already included.

Table 6 Group comparisons of symptomatology in CE vs. non-CE male patients

\begin{tabular}{|c|c|c|c|c|c|c|c|c|}
\hline & \multicolumn{4}{|l|}{ All boys } & \multicolumn{4}{|c|}{ Clinical group } \\
\hline & $\overline{\mathrm{CE}}$ & Non-CE & $t$ & $d$ & $\overline{C E}$ & Non-CE & $t$ & $d$ \\
\hline Age at onset & $14.0(1.45)$ & $13.7(1.75)$ & 0.77 & - & $13.9(1.50)$ & $13.7(1.68)$ & 0.63 & - \\
\hline \multicolumn{9}{|l|}{ EDEQ } \\
\hline Global & $3.0(1.37)$ & $1.8(1.59)$ & $4.13^{*}$ & 0.71 & $3.4(1.04)$ & $2.9(1.31)$ & $2.07^{*}$ & 0.46 \\
\hline Restraint & $3.0(1.64)$ & $1.7(1.84)$ & $3.97^{*}$ & 0.76 & $3.4(1.38)$ & $2.7(1.72)$ & 1.94 & - \\
\hline Binge $(M)$ & $3.9(2.71)$ & $7.0(7.99)$ & -1.76 & - & $4.2(2.72)$ & $6.3(4.47)$ & -1.53 & - \\
\hline \multicolumn{9}{|l|}{ SASB } \\
\hline Negative perfectionism & $38.6(23.39)$ & $25.6(24.06)$ & $2.92^{*}$ & 0.55 & $42.5(22.71)$ & $36.2(24.93)$ & 1.23 & - \\
\hline Self-esteem & $25.1(35.59)$ & $34.6(40.30)$ & -1.31 & - & $19.0(34.32)$ & $15.7(38.54)$ & 0.43 & - \\
\hline \multicolumn{9}{|l|}{ SDQ } \\
\hline Emotional distress & $4.6(2.68)$ & $3.4(2.49)$ & $2.65^{*}$ & 0.48 & $5.1(2.44)$ & $4.3(2.44)$ & 1.68 & - \\
\hline Hyperactivity & $4.6(2.20)$ & $3.5(2.66)$ & $2.49^{*}$ & 0.45 & $4.9(2.21)$ & $4.1(2.61)$ & 1.44 & - \\
\hline
\end{tabular}

See Table 1 for explanation of abbreviations

Note. CE compulsive exercise

* $=p<.05$ 
Some patients remitting from ED continued with CE, or began with $\mathrm{CE}$ during follow-up. $\mathrm{CE}$ is thus a common and problematic feature within EDs but not restricted solely to them, in line with other research findings $[11,23$, 38]. The most prominent feature of CE pathology within EDs, put forth by Boyd and colleagues [39], is withdrawal symptoms if not exercising, in the form of guilt and feeling annoyed or agitated. Emotional withdrawal symptoms of this kind may be exacerbated in ED specifically due to strict self-imposed rules for weight/shape control. This is suggested also by ED research on the association between $\mathrm{CE}$ and negative perfectionism $[6,18]$ and drive for thinness $[5,8]$, and also consistent with our findings. Negative reinforcement is also a driving force behind addictions [40]. As with other behaviors under the umbrella term of behavioral addiction, where $\mathrm{CE}$ has been suggested to be appropriately placed $[38,40]$, the activity itself is not detrimental (cf. sex, work, internet use, shopping), so abstaining is neither possible nor desirable. Instructing the patient to refrain from exercising altogether may alleviate $\mathrm{CE}$, but would be equivalent to 'throwing the baby out with the bathwater'; as the many physiological and psychological benefits of exercise would be lost [41]. In the acute phase of stabilization, restricting energy expenditure might be necessary. Over time however, clinicians may do well in helping the patient both to eat and to exercise for the right reasons, in a balanced and flexible manner, while also teaching adaptive emotion regulation strategies. A recent study has shown positive effects of supervised exercise on emotional state in adolescent inpatients with AN [42], although it remains to be seen if such interventions are beneficial for $\mathrm{CE}$ or the ED itself.

An important aspect to hold in mind as well is that care should be taken not to overpathologize exercise or other behaviors [43]. There might be a compulsive element to a behavior such as exercising but unless there is stability over time and clear functional impairment it does not classify as addiction [43]. Problematic over-involvement in exercise has been shown to be fairly transient in a 5-year cohort study, even when untreated [44]. Clinical guidelines on treatment of $\mathrm{CE}$ are however lacking, as are intervention studies specifically directed at $C E$. This would be a welcome next step of investigation.

\section{Denial of illness}

Denial falsely inflated differences between $\mathrm{CE}$ and non-CE patients. Despite receiving an ED diagnosis, 25\% of adolescents rated below clinical cut-off and self-report of CE was substantially lower among them (12\%), as was reporting of bingeing, purging and associated features. In this study, it was not possible to determine the underlying cause(s) behind denial, but the phenomenon clearly complicated assessment. Denial as a potential threat to validity should therefore be taken into account in studies on adolescents with EDs.

\section{Strengths and limitations}

As mentioned, a specific measure of CE was lacking and frequency estimates were based on recall, entailing inherent instability. Also, we did not measure duration of CE engagement, threatening the validity of the compulsivity aspect. A specific measure for negative perfectionism was also lacking. However, item content and theoretical underpinnings of SASB Self-criticism are consistent with the negative perfectionism construct, and results were consistent with previous research. The present study has several strengths as well: the large sample size, the longitudinal design, inclusion of understudied groups such as boys and EDNOS, inclusion of all levels of severity and controlling for denial. Patients were included nationwide, with high coverage ratio, ensuring high ecological validity. Well validated and structured methods for diagnostics and self-rating were used. An attrition rate of $48 \%$ is potentially a threat to generalizability, yet attrition analyses found no significant differences, in concordance with another study on the database [45]. Patients in the current study received treatment of various modalities (e.g., medical, psychological, pedagogical, nutritional, social, physical), length and intensity (e.g., inpatient, day-patient, out-patient). Although outcome was similar in $\mathrm{CE}$ and non-CE patients, it is not clear if they benefit equally from particular interventions, and this was not investigated. Recalling the instability of problematic behaviors such as exercise [44], assessment of the role of $\mathrm{CE}$ in the symptom pattern of the individual is warranted, as is attention to the possibility that patients may switch between CE and other compensatory/ addictive behaviors [46]. Clinically, different addictive or compulsive behaviors may be present interchangeably if the underlying function (emotion regulation) is not addressed.

\section{Conclusions}

About four in ten adolescents with ED report engaging in CE. Patients with CE have a more severe clinical presentation, albeit not affecting 1-year prognosis. Cessation of $\mathrm{CE}$ during follow-up was associated with remission. Systematic assessment of $\mathrm{CE}$ would aid in estimating its impact on ED, in tailoring treatment, and in evaluating outcome. Denial of illness is a common feature in adolescents with EDs and as such a potential confounder in research.

\section{Abbreviations}

CE: Compulsive exercise; ED: Eating disorder; EDEQ: Eating disorder examination questionnaire; MINI-Kid: Mini International neuropsychiatric interview for children and adolescents; SASB: Structural analysis of social behavior; SDQ: Strengths and difficulties questionnaire; SEDI: Structured eating disorder interview 


\section{Acknowledgements}

The study has been presented at The National Eating Disorder Symposium in Stockholm in February, 2016. We thank participants for valuable feedback.

\section{Funding}

The study was funded by the Stockholm County Council. The funding body had no influence on conception or execution of the study.

\section{Availability of data and materials}

The datasets generated during and/or analyzed during the current study are not publicly available due to reasons of confidentiality since it is a clinical sample and ethical requirements do not allow public sharing, but they are available from the corresponding author on reasonable request.

\section{Authors' contributions}

$C C$ and $A B$ performed a preliminary study on the topic. $J L$ expanded the study in aim and scope and was the major contributor for the current version of the manuscript. $A B$ and $J L$ jointly performed the analyses for the current version. All authors have read and approved the final manuscript.

\section{Competing interests}

The authors declare that they have no competing interests.

\section{Consent for publication}

Not applicable.

\section{Ethics approval and consent to participate}

Ethical approval was granted by the regional review board (Nr 2009/129831/1, EPN Stockholm).

Received: 5 September 2016 Accepted: 1 December 2016

Published online: 03 April 2017

\section{References}

1. Meyer C, Taranis L, Goodwin H, Haycraft E. Compulsive exercise and eating disorders. Eur Eat Disord Rev. 2011;19:174-89. doi:10.1002/erv.1122.

2. Kostrzewa E, Eijkemans M, Kas M. The expression of compulsive exercise cosegregates with the risk of developing an eating disorder in women. Psychiat Res. 2013;210:1123-8.

3. Davis C, Kennedy S, Ravelski E, Dionne M. The role of physical activity in the development and maintenance of eating disorders. Psychol Med. 1994;24:957-67.

4. Davis C, Katzman DK, Kaptein S, Kirsch C, Brewer H, Kalmbach K, et al. The prevalence of high-level exercise in the eating disorders: etiological implications. Compr Psychiatry. 1997;38:321-6.

5. Dalle Grave R, Calugi S, Marchesini G. Compulsive exercise to control shape or weight in eating disorders: prevalence, associated features, and treatment outcome. Compr Psychiatry. 2008:49:346-52.

6. Shroff H, Reba L, Thornton L, Tozzi F, Klump K, Berrettini WH, et al. Features associated with compulsive exercise in women with eating disorders. Int J Eat Disord. 2006;39:454-61.

7. Penas-Lledo E, Vaz Leal F, Waller G. Compulsive exercise in anorexia nervosa and bulimia nervosa: relation to eating characteristics and general psychopathology. Int J Eat Disord. 2002;31:370-5.

8. Solenberger S. Exercise and eating disorders: a 3-year inpatient hospital record analysis. Eat Behav. 2001;2:151-68.

9. Strober M, Freeman R, Morrell W. The long-term course of severe anorexia nervosa in adolescents: survival analysis of remission, relapse, and outcome predictors over 10-15 years in a prospective study. Int J Eat Disord. 1997;22:339-60.

10. Carter J, Blackmore E, Sutandar-Pinnock K, Woodside D. Relapse in anorexia nervosa: a survival analysis. Psychol Med. 2004;34:671-9.

11. Cook B, Engel S, Crosby R, Hausenblas H, Wonderlich S, Mitchell J. Pathological motivations for exercise and eating disorder specific healthrelated quality of life. Int J Eat Disord. 2014;47:268-72.

12. Smith $A$, Fink E, Anestis $M$, et al. Exercise caution: over-exercise is associated with suicidality among individuals with disordered eating. Psychiat Res. 2013;206:246-55.

13. Young S, Touyz S, Meyer C, Arcelus J, Rhodes P, Madden S, et al. Validity of exercise measures in adults with anorexia nervosa: the EDE, compulsive exercise test and other self-report scales. Int J Eat Disord. 2016. doi:10.1002/eat.22633.

14. Welch E, Birgegård A, Parling T, Ghaderi A. Eating disorder examination questionnaire and clinical impairment assessment questionnaire: general population and clinical norms for young adult women in Sweden. Behav Res Ther. 2011:49:85-91.

15. Mond J, Calogero R. Compulsive exercise in eating disorder patients and in healthy women. Aust N Z J Psychiatry. 2009;43:227-34.

16. Sternheim L, Danner U, Adan R, van Elburg A. Drive for activity in patients with anorexia nervosa. Int J Eat Disord. 2015;48:42-5.

17. Fietz M, Touyz S, Hay P. A risk profile of compulsive exercise in adolescents with an eating disorder: a systematic review. Adv Eat Disord. 2014;2:241-63.

18. Taranis $L$, Meyer C. Associations between specific components of compulsive exercise and eating-disordered cognitions and behaviors among young women. Int J Eat Disord. 2011;44:452-8.

19. Young S, Rhodes P, Touyz S, Hay P. The relationship between obsessivecompulsive personality disorder traits, obsessive-compulsive disorder and compulsive exercise in patients with anorexia nervosa: a systematic review. J Eat Disord. 2013;1:16.

20. Kiezebrink K, Campbell D, Mann E, Blundell J. Similarities and differences between compulsive exercising anorexia nervosa patients compared with DSM-IV defined anorexia nervosa subtypes. EWD. 2009;14:199-204.

21. Noetel M, Miskovic-Wheatley J, Crosby RD, Hay P, Madden S, Touyz S. A clinical profile of compulsive exercise in adolescent inpatients with anorexia nervosa. J Eat Disord. 2016;4:1.

22. Varnado-Sullivan P, Horton R, Savoy S. Differences for gender, weight and exercise in body image disturbance and eating disorder symptoms. EWD. 2006;11:118-25.

23. Goodwin H, Haycraft E, Willis AM, Meyer C. Compulsive exercise: the role of personality, psychological morbidity, and disordered eating. Int J Eat Disord. 2011;44:655-60.

24. Adkins EC, Keel PK. Does "excessive" or "compulsive" best describe exercise as a symptom of bulimia nervosa? Int J Eat Disord. 2005;38:24-9. doi:10. 1002/eat.20140

25. Lindstedt K, Neander K, Kjellin L, Gustafsson SA. Being me and being us adolescents' experiences of treatment for eating disorders. J Eat Disord. 2015;3:9. doi:10.1186/s40337-015-0051-5.

26. Birgegård A, Björck C, Clinton D. Quality assurance of specialised treatment of eating disorders using large-scale Internet-based collection systems: methods, results and lessons learned from designing the Stepwise database. Eur Eat Disord Rev. 2010;18:251-9.

27. de Man Lapidoth J, Birgegård A. Validation of the Structured Eating Disorder Interview (SEDI) against the Eating Disorder Examination (EDE). Stockholm: Socialstyrelsen; 2009.

28. Carter JC, Stewart DA, Fairburn CG. Eating disorder examination questionnaire: norms for young adolescent girls. Behav Res Ther. 2001;39:625-32.

29. White $H J$, Haycraft E, Goodwin H, Meyer C. Eating disorder examination questionnaire: factor structure for adolescent girls and boys. Int J Eat Disord. 2014:47:99-104. doi:10.1002/eat.22199.

30. Sheehan DV, Sheehan KH, Shytle RD, Janavs J, Bannon Y, Rogers JE, et al. Reliability and Validity of the Mini International Neuropsychiatric Interview for Children and Adolescents (MINI-KID). J Clin Psychiat. 2010;71:313-26.

31. Muris $P$, Meesters $C$, van den Berg $F$. The Strengths and Difficulties Questionnaire (SDQ)- further evidence for its reliability and validity in a community sample of Dutch children and adolescents. Eur Child Adoles Psy. 2003;12:1-8.

32. Benjamin L. Intrex User's manual. Salt Lake City: Univ Utah; 2000.

33. Pincus AL, Newes SL, Dickinson KA, Ruiz MA. A comparison of three indexes to assess the dimensions of structural analysis of social behavior. J Pers Assess. 1998;70:145-70.

34. Ekeroth K, Birgegård A. Evaluating reliable and clinically significant change in eating disorders: comparisons to changes in DSM-IV diagnoses. Psychiat Res. 2014;216:248-54.

35. Preti A, Rocchi MB, Sisti D, Camboni MV, Miotto P. A comprehensive metaanalysis of the risk of suicide in eating disorders. Acta Psychiat Scand. 2011; 124:6-17. doi:10.1111/j.1600-0447.2010.01641.x.

36. Josefsson T, Lindwall M, Archer T. Physical exercise intervention in depressive disorders: meta-analysis and systematic review. Scand J Med Sci Sports. 2014;24:259-72. doi:10.1111/sms.12050. 
37. Meyer C, Blissett J, Alberry R, Sykes A. Beliefs about exercise: relationship to eating psychopathology and core beliefs among young female exercisers. Eat Behav. 2013;14:79-82. doi:10.1016/j.eatbeh.2012.10.003.

38. Berczik K, Szabo A, Griffiths MD, Kurimay T, Kun B, Urban R, et al. Exercise addiction: symptoms, diagnosis, epidemiology, and etiology. Subst Use Misuse. 2012;47:403-17. doi:10.3109/10826084.2011.639120.

39. Boyd C, Abraham S, Luscombe G. Exercise behaviours and feelings in eating disorder and non-eating disorder groups. Eur Eat Disord Rev. 2007;15:112-8.

40. Sussman S, Lisha N, Griffiths M. Prevalence of the addictions: a problem of the majority or the minority? Eval Health Prof. 2011;34:3-56.

41. Hausenblas HA, Cook BJ, Chittester NI. Can exercise treat eating disorders? Exerc Sport Sci Rev. 2008;36:43-7. doi:10.1097/jes.0b013e31815e4040.

42. Noetel M, Miskovic-Wheatley J, Costa D, Ross D, Crosby RD, Hay P, Kohn M, Madden S, Touyz S. Exercise for the compulsive exercisers? An exploratory study in adolescent inpatients with anorexia nervosa. Adv Eat Disord. 2016:4:264-76

43. Billieux J, Schimmenti A, Khazaal Y, Maurage P, Heeren A. Are we overpathologizing everyday life? A tenable blueprint for behavioral addiction research. J Behav Addict. 2015;4:119-23. doi:10.1556/2006.4.2015.009.

44. Konkoly Thege B, Woodin EM, Hodgins DC, Williams RJ. Natural course of behavioral addictions: a 5-year longitudinal study. BMC Psychiatry. 2015;15:4. doi:10.1186/s12888-015-0383-3.

45. Ekeroth $\mathrm{K}$, Clinton D, Norring C, Birgegård A. Clinical characteristics and distinctiveness of DSM-5 eating disorder diagnoses: findings from a large naturalistic clinical database. J Eat Disord. 2013;1:31. doi:10.1186/2050-2974-1-31.

46. Haylett S, Stephenson G, Lefever R. Covariation in addictive behaviors: a study of addictive orientations using the shorter PROMIS questionnaire. Addict Behav. 2004:29:61-71.

\section{Submit your next manuscript to BioMed Central and we will help you at every step:}

- We accept pre-submission inquiries

- Our selector tool helps you to find the most relevant journal

- We provide round the clock customer support

- Convenient online submission

- Thorough peer review

- Inclusion in PubMed and all major indexing services

- Maximum visibility for your research

Submit your manuscript at www.biomedcentral.com/submit

) Biomed Central 